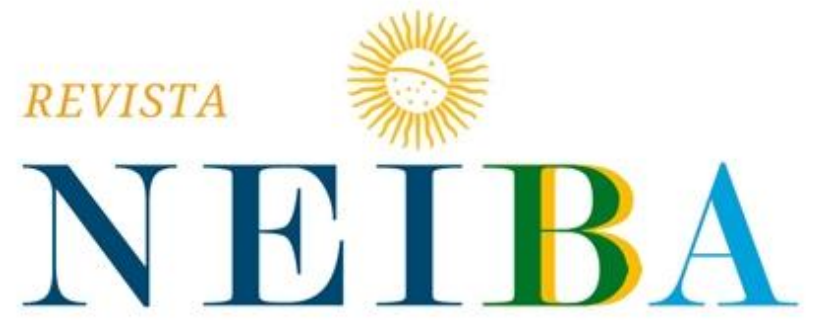

CADERNOS ARGENTINA-BRASIL
Volume 10, 2021, p. 01-33

DOI: 10.12957/neiba.2021.49932 | e49932 I ISSN: 2317-3459

\title{
LA INTERNACIONALIZACIÓN DE CIUDADES Y REGIONES EN LAS CONSTRUCCIONES TEÓRICAS DE LAS RELACIONES INTERNACIONALES
}

THE INTERNATIONALIZATION OF CITIES AND REGIONS IN THE THEORETICAL CONSTRUCTIONS OF INTERNATIONAL RELATIONS

\section{Carolina Pesuto ${ }^{1}$}

${ }^{1}$ Universidad Nacional de Rosario - Facultad de Ciencia Política y Relaciones Internacionales, Rosario, Santa Fe, Argentina. E-mail: caropesuto@gmail.com ORCID: https://orcid.org/0000-0002-7224-4827

Recebido em: 06/04/2020 | Aceito em: 05/01/2021. 


\section{RESUMEN}

El campo de estudio de la internacionalización de las ciudades y regiones ha ganado terreno en las relaciones internacionales de los últimos tiempos. El presente trabajo descriptivo se propone realizar una revisión de las construcciones teóricas de la disciplina de las relaciones internacionales que nutren los estudios sobre el tema. Intentaremos abordarlo describiendo aquellos aportes que los diferentes debates teóricos realizaron al fragmentado campo de estudios de la internacionalización subnacional. Revisaremos y describiremos los niveles de análisis que tratan el tema haciendo hincapié en cuatro subconjuntos dentro del campo de estudios: el debate sobre el actor internacional; la política exterior; la integración regional y el desarrollo y territorio en la internacionalización y ciudades y regiones. Todos ellos forman parte de las teorías de alcance medio que, bajo un eclecticismo teórico y pluralismo metodológico, actualmente hacen significativos aportes y categorías analíticas para la compresión del fenómeno multidimensional.

Palabras-clave: Relaciones Internacionales; Teoría; Gobiernos Subnacionales.

\section{SUMMARY}

The field of study of the internationalization of cities and regions has gained ground in international relations in recent times. The present descriptive work proposes to carry out a review of the theoretical constructions of the discipline of international relations that nourish the studies on the subject. We will try to address it by describing the contributions that the different theoretical debates made to the fragmented field of studies of subnational internationalization. We will review and describe the levels of analysis that address the issue, emphasizing four subsets within the field of studies: the debate on the international actor; foreign policy; regional integration and development and territory in internationalization and cities and regions. All of them are part of the medium-range theories that, under a theoretical eclecticism and methodological pluralism, currently make significant contributions and analytical categories for the understanding of the multidimensional phenomenon.

Keywords: International Relations; Theory; Subnational Governments. 


\section{1- INTRODUCCIÓN}

La comprensión del fenómeno de la internacionalización de ciudades y regiones a través de un recorrido por los debates teóricos de las relaciones internacionales (RRII) resulta atractivo teniendo en cuenta la multiplicidad de estudios surgidos sobre el tema en los últimos años en diferentes latitudes. Estos estudios han contribuido a relevar y visibilizar el comportamiento de estos actores y a preguntarnos sobre su relevancia o aporte a las RRII y a la política exterior.

El intento de enfocar el interés en aquellos debates y teorías de RRII que aportan al campo de estudios sobre el tema constituye un desafío interesante debido a la fragmentación que se observa en dicho campo y a la heterogeneidad y complejidad de aspectos que involucra la proyección internacional de gobiernos subnacionales ${ }^{2}$. Las explicaciones del fenómeno han sido elaboradas en combinación con otros campos de estudio entre los cuales se pueden mencionar la economía política, la teoría del desarrollo y la toma de decisiones entre otras.

Un gran número de investigaciones han abordado la descripción y caracterización del accionar internacional de los gobiernos subnacionales lo que ha constituido un notable avance en la temática. De esta manera estos estudios permiten conocer quiénes (gobiernos provinciales, regionales o municipales) han implementado estas estrategias; dónde (Norteamérica, Europa, América Latina) se concentran la mayor cantidad de experiencias; y cómo (relaciones bilaterales, multilaterales, políticas locales de comercio exterior, marketing de ciudades) han llevado adelante este tipo de estrategias (Duchacek, 1990; Michelmann y Soldatos, 1990; Colacrai y Zubelzú, 1994 y 2004; Garcia Segura, 1996; Zapata Garesché, 2007; Bastista, Jakobsen y Evangelista, 2008; Maira, 2010; Schivavon, 2010; Vigevani y Prado 2010; Salomon, 2007; Calvento, 2016). Para analizar estos procesos la disciplina revisó marcos conceptuales existentes y se generaron nuevos términos como el de paradiplomacia, ampliamente utilizado como abreviación de "diplomacia paralela" (Michelmann y Soldatos, 1990). La terminología

\footnotetext{
${ }^{2}$ En el presente trabajo se tomará como sinónimo el término subnacional y subestatal; haciendo referencia al involucramiento de entidades políticas menores que el Estado o no centrales, que pueden ser ciudades o gobiernos locales así como gobiernos provinciales o regionales, que actúan en RRII con diversos objetivos y motivaciones. Para ampliar el tema se recomienda García Segura (1996); Cornago Prieto (1999, 2010); Maira (2010).
} 
para dar cuenta de dicho fenómeno es motivo de discrepancia o falta de consenso dentro del fragmentado campo de estudios de la subestatalidad en las RRII ${ }^{3}$.

El presente trabajo se propone realizar una revisión de las construcciones teóricas de la disciplina de las RRII que nutren los estudios sobre el tema. Tiene como objetivo describir aquellos debates teóricos que mayormente aportaron al fragmentado campo de estudios de la internacionalización subnacional haciendo hincapié en los niveles de análisis y en cuatro subconjuntos de miradas sobre el tema: el debate sobre el actor internacional; la política exterior; la integración regional y por último aquellos que lo abordan desde el desarrollo y territorio.

Primeramente describiremos los lineamientos del "tercer" debate en el desarrollo teórico de las RRII; destacando las obras sobre interdependencia compleja y el transnacionalismo que han contribuido con categorías valiosas para comprender nuevos actores y nuevos temas que surgieron en el sistema internacional. Luego retomaremos algunos argumentos del debate entre neorrealistas y neoliberales (neo-neo); sin ahondar en el cuarto debate; solamente rescataremos algunos elementos que han aportado e influido en los estudios sobre paradiplomacia. A continuación se presentarán algunas consideraciones sobre la vuelta del Estado y la política, y de cómo las perspectivas teóricas enmarcan una visión cooperativa y coordinada de la internacionalización de gobiernos subnacionales y el gobierno central. Por último, revisaremos la temática sobre el nivel de análisis y las teorías de alcance medio que nutren los estudios sobre subestatalidad en los últimos tiempos organizando nuestra exposición en cuatro subgrupos de enfoques.

En nuestro tiempo el eclecticismo teórico y las combinaciones de enfoques son las que promueven mejores comprensiones y preguntas sobre el campo de internacionalización de gobiernos locales y regiones en las RRII. Lo anterior deja de lado la adscripción a una única corriente teórica como abordaje completo de fenómenos complejos como el que nos aboca; sino que adscribimos a un pluralidad y tolerancia

\footnotetext{
${ }^{3}$ Se han propuesto variadas denominaciones sobre el fenómeno tales como: política internacional subnacional; gestión, proyección y participación internacional, también se mencionan vinculación externa y acción exterior entre otras tantas. Fueron Duchacek (1990) y Michelmann y Soldatos (1990) quienes crearon el término paradiplomacia, poniendo énfasis el prefijo "para" a fin de dar cuenta de la capacidad de los gobiernos locales en llevar adelante una diplomacia "paralela", es decir, "subsidiaria o accesoria" respecto a los gobiernos centrales. Para ampliar el tema se recomienda Calvento (2016) y Zeraoui (2016).
} 
entre teorías y sus variadas construcciones analíticas que sirvan de andamiajes para abordar sobre la temática (Lake, 2013).

Por último, cabe mencionar que el presente artículo es llevado adelante sobre la base de una estrategia metodológica cualitativa, con revisión de fuentes bibliográficas y publicaciones académicas y científicas de la disciplina. Siendo un trabajo de tipo teórico, la técnica de construcción de datos y argumentaciones es de tipo documental y de revisión de contenido.

\section{2- LAS PERSPECTIVAS TEÓRICAS EN EL DEVENIR DE LA DISCIPLINA}

Todas las teorías y las disciplinas académicas responden en parte a los sucesos que ocurren en el mundo real dirá Halliday (2006); quien considera que el auge en los estudios de las RRII es un producto del cambio en tres círculos concéntricos: uno de la propia disciplina y sus dinámicas; otro de las ciencias sociales en los que se inserta y un tercero refiere a los sucesos de la realidad que la alimentan.

Las RRII comparten con otras ciencias una tradición de debates, la historiografía de la disciplina puede comprenderse a través de "grandes debates" a través de los cuales los intentos de diálogo, reflexiones, renovaciones y la aparición de fracturas teóricas cuestionan los supuestos básicos de las teorías precedentes (Sodupe, 2003). En este sentido, es posible mencionar cuatro grandes debates, aclarando que esta construcción es a los fines de la comprensión del proceso ya que algunos de ellos no se concretaron en diálogos y contrapuntos reales efectivamente publicados (Salomón González, 2002).

Primeramente surgió en las RRII el debate clásico entre idealismo y realismo, estas tradiciones de pensamiento dominaron ampliamente la disciplina abarcando temporalmente desde la primera guerra mundial; se destacan su visión sobre el poder, la centralidad del actor estado y las dinámicas del sistema internacional serán de mucha influencia. Un segundo debate enfrentó a tradicionalistas (idealistas y realistas) con los cientificistas durante los años '50 y '60 con un eje de debate de tipo metodológico, los tradicionalistas utilizaban técnicas de análisis de carácter histórico, descriptivo e intuitivo mientras que los cientificistas estaban influidos por la revolución behaviorista en las ciencias sociales centrada en la medición y las metodologías cuantitativas logrando explicaciones sobre evidencia empírica. 
El tercer debate es el denominado debate inter-paradigmático cubrió los dos decenios siguientes; se dio entre tradicionalistas y los transnacionalistas o globalistas. El cuarto debate supuso la confrontación entre racionalistas y reflectivistas surgió con fuerza en los '90 y continúa en los primeros años del siglo XXI, este último no será abordado en profundidad en el presente trabajo (Sodupe, 2003:16).

A continuación se destacarán aspectos tanto del tercer entre el realismo y el liberalismo o transnacionalistas; y luego se mencionarán argumentos del debate entre neorrealismo/neoliberalismo (racionalistas) y reflectivistas. Dicho recorte responde a los fines de comprender cómo las renovaciones teóricas en disciplina de las RRII nutren la observación del fenómeno de la internacionalización subnacional y las transformaciones de las últimas décadas del siglo XX en cuanto a actores, conexiones y dinámicas del sistema.

Las transformaciones que anteceden y son propulsoras del incremento en la intensidad y extensión del accionar internacional de gobiernos subnacionales estuvieron ligadas a los cambios de la realidad mundial. Por consiguiente, la teoría de las RRII, como campo de pensamiento, ha evolucionado en paralelo a los cambios ocurridos en el sistema internacional. Estos cambios producen reacomodamientos dentro de los paradigmas de las RRII, sostiene Celestino del Arenal, en especial entre el paradigma tradicional, realista o estatocéntrico, que se reconoce como preponderante y aquel de la sociedad global, transnacionalista o de la interdependencia. Con la aparición de nuevos actores, factores, problemas y objetivos que transforman la realidad internacional resultan creaciones a nivel teórico. Dicho autor reconoce que una tendencia hacia la reconciliación, la complementariedad o el pluralismo teórico entre los distintos paradigmas. La compleja realidad internacional, impide negar tanto la importancia de los Estados como de los actores transnacionales, tanto de las relaciones interestatales como de las relaciones transnacionales, tanto de situaciones de conflicto como de cooperación, hace que la teoría de las RRII haya optado, en cierta medida por una solución ecléctica y de compromiso (1989: 179).

La teoría de la interdependencia compleja y las relaciones transnacionales de Robert Keohane y Joseph Nye (1988), así como las propuestas de James Rosenau (1990) 
se enmarcan en el tercer debate. Suele caracterizarse como el debate interparadigmático entre autores realistas y los defensores de concepciones de la sociedad global, transnacionalistas o de la interdependencia. El mismo estuvo marcado por la aparición en el campo de las RRII de una reacción frente a las observaciones y a los métodos propuestos por el realismo para comprender la realidad del sistema internacional ${ }^{4}$.

Dicho debate estuvo enmarcado por el contexto político de los '70 caracterizado por la distensión, la crisis del sistema de Bretton Woods, crisis del petróleo y el aparente declive de la hegemonía estadounidense en el sistema internacional (Keohane, 2012). El tránsito de un sistema bipolar rígido a otro más flexible, desjerarquizado y complejo puso en cuestionamiento los conceptos centrales del pensamiento realista. En este nuevo escenario, aparecieron nuevas categorías: multipolaridad, distensión, interdependencia, transnacionalización (Russel, 1986). Las perspectivas transnacionalistas o interdependentistas en el campo disciplinar implicaron una ruptura paradigmática puesto que cuestionó los basamentos de las relaciones internacionales: 1) las imágenes del mundo, 2) los actores esenciales y/o unidades de análisis, 3) las causas de la guerra y de la paz y, subsiguientemente, la naturaleza del poder. La mirada transnacionalista en las RRII no se corresponde con el modelo exclusivamente conflictivo del enfoque realista, sino que responden a un modelo que plantea un mayor margen de maniobra para los Estados. En este sentido, la cooperación internacional es posible y es un modo de atenuar la situación de anarquía. Robert O. Keohane y Joseph Nye fueron los responsables de las dos obras más significativas de la corriente transnacionalista: Transnational Relations and World Politics (1971) y Power and Interdependence (1977).

Dichos autores sostuvieron que la estructura del sistema internacional respondía a una matriz que denominaron de "interdependencia compleja", la cual reunía una serie de características distintivas. En primer lugar, reconocen una multiplicidad de canales de conexión entre las distintas sociedades nacionales. Por lo tanto, a las clásicas relaciones

\footnotetext{
${ }^{4}$ Fue a partir de la introducción en las RRII de la noción kuhniana de "paradigma científico" que el tercer debate puede ser definido como "debate interparadigmático" entre el paradigma realista, el transnacionalista (o globalista, o liberal, o pluralista, o de sociedad mundial, según los autores) y el estructuralista (también definido como marxista aunque no todos los autores estructuralistas se consideran marxistas- 0 , complicando aún más las cosas, como globalista), por más que la participación de las corrientes marxistas fue muy restringida (Del Arenal, 1984; Salomón, 2002).
} 
interestatales -consideradas por idealistas y realistas-, debían sumarse las relaciones transnacionales, esto es, las que se producen entre actores privados, y las relaciones transgubernamentales, que son aquellas que tienen lugar entre agencias gubernamentales pertenecientes a distintos países. Estos aportes serán esenciales para enmarcar la actuación exterior de los gobiernos subnacionales en las RRII.

A la multiplicidad de canales de conexión se sumaba la proliferación de actores ocurrida en el escenario global. El Estado había dejado de monopolizar el manejo de las relaciones externas mientras que actores no gubernamentales habían comenzado a intervenir en ellas debido al proceso de transnacionalización. Asimismo, la interdependencia compleja daba cuenta del fenómeno de la expansión internacional de la sociedad civil; integrando la irrupción de ONGs como agrupaciones constituidas para la persecución de determinados valores o intereses.

La matriz de interdependencia compleja se caracterizaba por la ausencia de jerarquías entre las cuestiones que componen la agenda internacional; los aspectos vinculados a la seguridad militar no dominaban necesariamente la agenda y no existe la división entre "alta política" y "baja política". De este modo, a las agendas de seguridad y geopolítica se añaden otras agendas que incluyen temas como relaciones económicas y culturales, desarrollo y el subdesarrollo, desigualdad y hambre, agotamiento y explotación de los recursos, desequilibrio ecológico y violación de derechos humanos. En consecuencia, existe una clara conexión entre las cuestiones de política interna y las de política exterior.

Para Keohane y Nye el poder dejaba de ser medido solamente en términos de la acumulación de capacidades militares y será concebido en términos de influencia, lo cual requiere ser contextualizado en un área de cuestiones (issue area) a fin de considerarlo poder político, económico o militar, entre otros. La interdependencia compleja era definida como una situación caracterizada por efectos recíprocos entre países o actores de diferentes países. Pero a pesar de los costos recíprocos ambos reconocían que había países que tenían más costos que otros, al punto de que la interdependencia se podía trasformar en dependencia o en situaciones extremas para los países más vulnerables (1988: 22). 
Si bien los autores mencionados no abordaron la cuestión de internacionalización de gobiernos locales en sus obras; se reconoce que sus contribuciones han posibilitado una apertura del campo disciplinar. Los avances teóricos con el reconocimiento de los canales transgubernamentales en las RRII, como las redes de relaciones entre burocracias gubernamentales, posibilitaron líneas de análisis sobre los actores gubernamentales domésticos o no centrales, a partir de las cuales comenzarían los estudios sobre la acción internacional de los gobiernos subnacionales.

A principios de la década del ochenta se abre un nuevo debate entre racionalistas (neorrealistas y neoliberales) con los reflectivistas ${ }^{5}$. Sodupe sostiene que este "es un debate que se enmarca en lo que se denomina crisis de la modernidad o del proyecto surgido en la ilustración en la cual entroncan los aportes racionalistas" (1993:18). Solamente mencionamos este debate ya que vuelve a redefinir el campo de la disciplina, lo cual decantó en categorías de análisis que se incluyeron en los estudios sobre internacionalización de ciudades y regiones en las RRII. A los fines del presente trabajo no es necesario ahondar en el cuarto debate, ya que el tercer debate permite entender las diferencias entre concepciones estatocéntricas y globalistas que se reconvirtieron en neorrealismo y noeliberalismo, y pasaron a compartir un programa de investigación "racionalista" en el cuarto debate.

El eje central del debate neo-neo refiere a cuáles es el papel de las instituciones internacionales y los regímenes en el comportamiento de los Estados en una situación de anarquía internacional. La pregunta central que nutre el intercambio es si las instituciones internacionales pueden o no superar los efectos de la anarquía en el sistema internacional.

Para los neoliberales las instituciones y regímenes mitigan los efectos constreñidores que tiene la anarquía sobre la cooperación, mientras que los neorrealistas consideran exagerado el papel que atribuyen los neoliberales a regímenes e instituciones. Queda en evidenciado que las creencias de cada grupo están presentes ancladas en las tradicionales visiones del mundo realista y liberal. Ambos grupos de

\footnotetext{
5 El debate neo-neo puede considerarse también el "último gran debate" entre racionalistas y reflectivistas (Lake 2013: 570). Entre los reflectivistas se incluyen valiosos aportes del constructivismo (Adler, 2013; Wendt, 1987), del postmodernismo (Ashley, 1986; Devetak 1996), de la teoría crítica (Brown, 1994; Linklater, 1992) y del feminismo (Enloe, 1990; Tickner, 1992) en Waever (1996) y Lake (2013).
} 
autores realizan sus investigaciones con rigurosidad intentando demostrar sus ideas y darles validez a sus supuestos a partir de la confrontación de teorías y de hechos (Salomón, 2002: 17).

Durante los primeros años de la posguerra fría, al desaparecer los obstáculos derivados de la competencia entre Washington y Moscú, los neoliberales observaron mayores márgenes de viabilidad para la cooperación en el sistema internacional, y que las normas e instituciones democráticas limitaban el recurso a la guerra y mantenían la paz entre los Estados. Percibían que los Estados autoritarios, no democráticos, tendían mayoritariamente al uso de la violencia, por lo cual se instaba a repensar en un rol más preponderante para Naciones Unidas para el logro de pacificación del sistema.

En otro sentido, la postura neorrealista respecto a los beneficios de la cooperación se basaba en que los Estados cooperan buscando mejorar su posición relativa frente a los demás. En cuanto a las prioridades de las metas estatales ambos enfoques entienden que tanto la seguridad como el bienestar económico son metas importantes, aunque los neorrealistas, igual que los realistas clásicos, ponen el énfasis en la seguridad. En tanto los neoliberales consideran que las prioridades económicas son básicas para los Estados.

Los neorrealistas consideran que la distribución de recursos (capacidades) de los Estados es el factor que mejor explica su comportamiento. Para los neoliberales los regímenes y las instituciones disminuyen los efectos negativos que tiene la anarquía sobre la cooperación. Sin negarlo, los neorrealistas son críticos respecto del papel que atribuyen los neoliberales a regímenes e instituciones.

El dialogo neo-neo se ha plasmado fructíferamente en la subdisciplina de la Economía Política Internacional (EPI) y ha aportado a los estudios sobre subestatalidad en particular en aquellos abordajes que relacionan internacionalización con el desarrollo y el territorio (Strange, 1988; Boisier, 2010). Asimismo, el concepto de régimen internacional $^{6}$ es otro valorado producto del debate neo-neo aportado por Krasner (1982) y empleado en todas las corrientes teóricas de la disciplina (Salomón González,

\footnotetext{
${ }^{6}$ Un régimen internacional es un conjunto de principios, implícitos o explícitos, normas, reglas y procedimientos de decisiones alrededor de los cuales las expectativas de los actores convergen en una determinada área de las RRII (Krasner, 1983).
} 
2002). Se puede rastrear la influencia de dicho concepto en los estudios sobre internacionalización de ciudades y regiones dentro de asociaciones y redes de gobiernos locales que existen a nivel regional y global; así como en aquellos que abordan interacciones de actores subestatales con las agendas globales (Zapata Garesché, 2007).

Los estudios sobre la cooperación internacional al desarrollo, son los que plasman el concepto de régimen internacional, ya que así lo entienden y analizan su constitución como una importante herramienta al servicio de la internacionalización de ciudades y poderes locales a través de la modalidad de cooperación descentralizada. Estos estudios sobre cómo los gobiernos subnacionales acceden a cooperación internacional, realizan aprendizajes y desarrollan capacidades de relacionamiento y vinculación internacional se destacan en la producción de los últimos años (Romero, 2004; Batista et. al, 2008; Martin, 2010).

Las relaciones surgidas de la globalización a partir de la posguerra fría son cada vez más complejas porque involucran múltiples niveles que dan origen a lo supranacional y a lo subnacional. En este contexto el concepto de soberanía es revisado en las RRII y empieza a mostrar límites en las nuevas dinámicas mundiales. Como emergentes de lo anterior podemos mencionar la proliferación de los acuerdos regionales de integración y la firma de tratados de libre comercio (TLC) en todo el planeta, la aparición de entes y redes internacionales, el peso de las grandes corporaciones transnacionales, la creciente movilización y visibilidad de actores de la sociedad civil en torno de temas y problemas de alcance global y fuerte impacto local, así como la emergencia de las llamadas ciudades-regiones y aún de ciudades globales y actores subnacionales, cada vez más autónomos en su proyección internacional. Todas ellas constituyen tendencias que erosionan las formas tradicionales de soberanía estatal tanto para actuar internamente como para abordar los asuntos externos o mundiales.

Desde la perspectiva de Halliday, el argumento sobre la desaparición del Estado y sobre la globalización no ofrece un consenso sobre el surgimiento de un nuevo sistema internacional, sino que más bien ofrece un nuevo contexto para explorar procesos contradictorios que conlleva esta globalización selectiva con implicancias diversas (2006: 17). 
Durante esos años el debate sobre el Estado como actor internacional tuvo dos ejes: la funcionalidad para organizar la actividad humana en un mundo globalizado, y la dispersión creciente de su poder hacia otras estructuras de autoridad sub y supranacionales. Este proceso será denominado de diversas maneras, en el caso de Bull lo llamará "neomedievalismo" mientras que Duchacek lo denominó "perforación de soberanía" (citados en Russell 2010: 88).

En aquellos años, el nuevo orden internacional de posguerra fría generó observaciones y teorizaciones que reflejarán los cambios tanto en la formulación de políticas públicas internacionales, como en las relaciones entre los diversos actores estatales, donde la supremacía de las grandes potencias sería reemplazada por relaciones horizontales, de cooperación y complementación de intereses, orientadas a resolver problemas globales. De esa forma, se iría reduciendo el monopolio de las grandes potencias en las RRII y se crearían un sistema de autoridad colectiva, ejercida por múltiples niveles gubernamentales, que incluyan a sociedad civil, academia y empresas, en la que prevalezcan relaciones de reciprocidad asociadas con la obtención de beneficios equivalentes. Estas nuevas formas de multilateralismo global y la diseminación de redes transnacionales, muchas de ellas creadas por los gobiernos subnacionales posibilitarán las visiones respecto a un nuevo orden internacional basado en la interdependencia entre los distintos niveles de gobierno (Luna Pont, 2011:80).

En los años 2000 se observa un retorno del Estado y de la política en los debates de las ciencias sociales en general y en las RRII en particular, caracterizado como un tiempo de reflexión que se abrió a partir de los atentados terroristas de septiembre de 2001 en Estados Unidos. Según Russell dicho debate se manifestó en tres planos; por un lado la unipolaridad y el papel del sistema de Estados; el tema de los "Estados fallidos" y "canallas", y por último la reconstrucción del Estado, en gran parte del mundo subdesarrollado en su rol de potenciador de las políticas públicas (2010: 88).

Con la crisis del capitalismo de 2008 se retoma la discusión en torno a la relación entre Estado y mercado, y cuál sería el rol de la política en un mundo interdependiente que produce demandas crecientes de bienes públicos globales, quienes los producen y los sostienen. En este nuevo contexto, los estudios de RRII sobre la relación entre el 
Estado nación y los gobiernos subnacionales denotan un carácter cooperativo en la relación. Ya no se piensa en enfatizar la diferencia y la oposición de intereses, sino que se subraya la necesidad de lograr una articulación positiva entre ambos actores.

En este sentido Russell sostiene la preponderancia de una coordinación constructiva entre la diplomacia nacional y la paradiplomacia o acción internacional de actores subnacionales (2010:98). Resulta imperioso que lo actores subnacionales lleven adelante su accionar internacional con una la lógica de complementación con lo Estados centrales; lo cual adquiere un carácter estratégico en un contexto mundial que ofrece numerosas oportunidades pero también nuevos desafíos. Problemáticas relevantes como las migraciones, pobreza, deterioro ambiental y crimen organizados con consecuencias en la seguridad pública e internacional reclaman una creciente cooperación tanto entre como dentro de los Estados.

En las RRII actuales se reconoce una proliferación de estudios basados en el eclecticismo teórico que se nutren de los intersticios que surgen sobre el final del tercer debate (interparadigmático y epistemológico) y aquellos aportes del dialogo neo-neo. Según Lake las teorías de alcance o nivel medio, que comparten tanto el positivismo como la perspectiva internacionalista en sus estudios de política, son las que proliferan en la actualidad (2013: 570).

El objetivo de las teorías de alcance medio es explicar particularidades respecto a la política exterior de los Estados y cómo se producen diferentes reacciones en cuanto al plano internacional en países diversos ante los mismos estímulos. Lo anterior incluye la revalorización de lo doméstico en las RRII y produjo un campo fértil para incorporar estudios de economía política internacional (EPI). Estos estudios se caracterizan por el pluralismo metodológico, acercamientos desde la historia y desde el estudio de casos así como abordan grandes conjuntos de datos en sus observaciones. Priorizan la contingencia histórica y focalizan sus estudios en una parte del proceso político, no intentan abarcarlo en su totalidad y estudian los efectos de una o varias variables de decisión política así como sus resultados ${ }^{7}$.

\footnotetext{
${ }^{7}$ Entre los autores pioneros en estudios en base a teorías de alcance medio podemos mencionar a investigaciones sobre política burocrática de Allison (1971); opinión publica de Hosty y Rosenau (1984), instituciones políticas de Waltz (1967) y estructuras de estado de Katzenstein (1978) entre otros.
} 


\section{3- NIVELES DE ANÁLISIS Y REVISIÓN DE SUBCONJUNTOS DE TEORÍAS}

Si bien compartimos con Lake (2013) que en los últimos años el eclecticismo teórico y la pluralidad metodológica son las fuentes más prolíferas de progresos en la disciplina, para diferenciarse de las posiciones y argumentos suscitados en los denominados "grandes debates" de las RRII. Es importante reconocer aquellos aportes que el tercer debate teórico y el intercambio neo-neo han realizado al fragmentado campo de estudios de la internacionalización subnacional. Ya se ha mencionado, que el debate entre realistas y el transnacionalismo/interdependencia compleja ha contribuido con innovaciones conceptuales que amplían los marcos de análisis sobre el fenómeno bajo estudio a pesar de no abordar la subestatalidad en forma directa. El recorrido por el debate neo-neo ha permitido identificar enfoques como la EPI y en el de régimen internacional que aportan a la comprensión de la internacionalización de gobiernos locales. En apartados sucesivos se describirán los abordajes que relacionan internacionalización con desarrollo y territorio, así como los que revisan la participación de actores subnacionales en asociaciones y redes de ciudades a nivel global.

Resulta pertinente entonces indagar y describir los niveles de análisis que mejor aborden la cuestión subnacional en las RRII, el qué, quiénes y cómo en la temática que serán presentadas a continuación en teorías de alcance medio.

Las primeras posturas teórico-metodológicas para el estudio de los actores subnacionales en RRII datan de finales de los '70 a los '80. Ellos centran sus abordajes en una concepción transnacionalista, tomando la unidad de análisis (gobiernos subnacionales locales o regionales) junto a otros actores no estatales. Según Lara Pacheco el estudio de las ciudades en las RRII no es nuevo, e identifica como primer artículo publicado el de Kenneth Boulding de 1968 "The City as an Element in the International System", quien centra su mirada sobre las epatas históricas que transitó la ciudad en el sistema internacional e hipotetiza sobre su posible desaparición en una completa ciudad-mundo (2019:78).

Sin embargo la proyección internacional de los actores subnacionales se dio con mayor intensidad a partir de la posguerra fría en el marco de la lucha por atraer inversiones y posicionarse en los mercados mundiales. El fenómeno de subestatalidad 
no es novedoso, ya que tanto Soldatos (1990) como Paquin y Lachapelle (2005) estudiaron el rol de los Estados federados y las unidades subnacionales en el caso de Quebec, Canadá remontándose hasta 1882. La particularidad de las últimas décadas es que han aumentado exponencialmente tanto el volumen como la velocidad de las interacciones de unidades subnacionales, el número de socios y la diversidad de temas de agenda implicados en las RRII (Ippolito, 2017).

Entre los aportes referidos a los niveles de análisis Singer (1961) refiere primariamente a los agregados o entidades sociales y a su estudio a fin de describir, explicar o predecir como puntos de análisis en política internacional. Russell define los niveles de análisis como puntos ordenadores en cuanto al tamaño y la complejidad teniendo en cuenta los actores - sea Estados o individuos-, sus acciones, las influencias que soportan y las explicaciones que queremos dar sobre ellas (Lee Ray, 2001:356).

Desde la publicación del trabajo de Snyder (1962) el estudio de la política exterior pasó a considerar el plano doméstico, en particular el proceso decisorio, como variable explicativa de los comportamientos de los estados en el plano internacional (citado en Gámez 2005:138). A partir de allí los abundantes estudios sobre análisis de la política exterior (APE) aportaron la idea de que los actores y factores domésticos son relevantes para el contenido y la formulación de la política externa y que dependería del "cuando" (el contexto histórico) y el "donde" (el área temática específica) para definir su peso como variable explicativa.

Las líneas de investigación que abordan las causas de la internacionalización de entidades subnacionales se pueden clasificar desde una doble perspectiva. Por un lado, algunas explicaciones ponen el acento en la dinámica y estructura del sistema internacional, que podría jugar como variable dependiente. Por otro lado, los análisis y estudios sobre política exterior que hacen hincapié en las estructuras o factores internos que impulsan el accionar internacional de actores subnacionales (Maira, 2010). Estudios que toman en cuenta el orden doméstico, el régimen de gobierno y el sistema político interno de las naciones; como Duchacek (1990) y Soldatos \& Michelmann (1990); en estudios sobre países centrales son ejemplos de esta última corriente. 
La forma en que ha sido abordada la internacionalización de ciudades y regiones en la disciplina responde a un esfuerzo de producción y sistematización de diversos aportes surgidos en los últimos tiempos, que está muy lejos de ser conclusiva sino en continuo desarrollo. Entre ellos se destacan los trabajos realizados por García Segura (1996); Colacrai y Zubelzu (1994, 2004); Cornago (1999, 2010); López y Oddone (2010); Luna Pont (2010); Maira (2010); Calvento (2016) y Oddone (2016) que serán retomados y reseñados en el siguiente apartado.

A los fines de ordenar nuestra presentación nos valemos de cuatro subconjuntos de enfoques de la subestatalidad en las RRII: a) aquellos centrados en la perspectiva del actor internacional; b) aquellos que lo abordan desde el análisis de políticas exteriores (APE) y los procesos de toma de decisiones; c) desde las teorías de la integración regional y, finalmente d) perspectivas que articulan la relación entre territorialidad y el

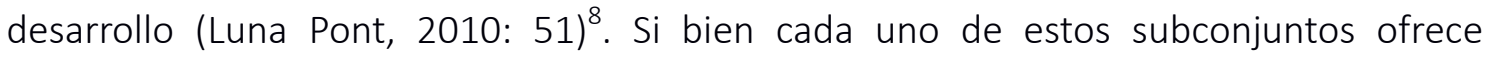
supuestos de partida y ejes analíticos distintos los límites entre unos y otros son ambiguos y ofrecen vasos comunicantes entre distintas aproximaciones.

\section{A - Enfoques Centrados En Las Perspectivas Del Actor}

En la disciplina predominan las visiones estatocéntricas, fundamentalmente jurídicas y formales. La transformación en la estructura del sistema internacional que supuso la postguerra fría impactó en la centralidad del Estado generando transformaciones en una doble dirección: hacia arriba y hacia abajo. Hacia arriba con la construcción de la arquitectura supranacional con la intensificación de los procesos de integración; hacia abajo creció el activismo internacional de los actores subnacionales (Maira, 2010: 17).

La globalización se convierte en el denominador común de las explicaciones sistémicas sobre paradiplomacia. La nueva oleada de acuerdos regionales de integración, la emergencia de las llamadas ciudades-regiones y aún de ciudades globales y de regiones que comenzaban a tener por sí mismas una importante proyección internacional, fueron percibidas como rasgos que se profundizarían y reflejarían un

\footnotetext{
${ }^{8}$ Esta división ha sido presentada primeramente como diferentes perspectivas sobre el tema por Luna Pont (2010 y 2011), en el presente trabajo ha sido retomada y enriquecida.
} 
mundo que es descrito como una aldea global, policéntrica y de soberanías perforadas en los que las definiciones territoriales serán revisadas, y a su vez transformaban las vinculaciones intergubernamentales, los patrones productivos y comerciales, las formas de inserción internacional y la sociedad global.

Según Lara Pacheco, las perspectivas teóricas que revisan a los gobiernos subnacionales como agente en las RRII se dieron fuertemente en la década de 2010. Dicho auge en los estudios teóricos-conceptuales sobre paradiplomacia tuvo como objetivo comprender la interacción entre la gobernanza global y las ciudades (globales) mientras que otros se han centrado la importancia de la identidad y su influencia a nivel exterior (2019:108).

En dicho contexto socio histórico global vemos instalarse el paradigma de governance, que es utilizado para indicar cambios en la forma en que se construyen las políticas y las formas de acción colectiva. En el proceso de incorporación de nuevos actores la sociedad global aparece como un elemento que debe ser tenido en cuenta. Esta nueva sociedad internacional y cosmopolita influye en el contexto internacional y en la composición de las RRII y aparece además un nuevo elemento, que es la valorización del territorio y de lo local, que toma forma a través del nuevo localismo y al papel de las ciudades (Marx, V., 2010: 28).

Lo anterior alude a un nuevo estilo de gobierno, como modalidad de coordinación no jerárquica, caracterizado por un mayor grado de cooperación e interacción entre el Estado y actores no estatales al interior de redes decisionales mixtas entre lo público y lo privado, nacionales e internacionales, orientadas a soluciones colectivas de problemas. Remite en forma primaria a la relocalización y desagregación de la autoridad gubernamental antes centrada en el Estado-nación hacia otros niveles en una dimensión vertical (supra: corporaciones, organismos y regímenes internacionales, e infra: actores subnacionales) y horizontal (Zubelzú, 2008). Los enfoques desde la governance apuestan a reconstruir fenómenos, conceptos y teorías que consideraban la elaboración de las políticas públicas como la acción exclusiva de los gobiernos centrales a través de un proceso racional y jerarquizado (Marx, V., 2010). 
Siguiendo a Zapata Garesché (2007) el concepto de actor político subestatal refiere a instancias socioterritoriales menores al Estado nación que pueden ser de distintos tipos incluyendo a ciudades y regiones ${ }^{9}$. El concepto se completa con los aportes de García Segura, quien los caracterizan por ser territoriales, porque son entidades que tienen una base física precisa. También son gubernamentales, y unidades constituyentes de un Estado compuesto (federación o confederación) o de un Estado unitario con una administración territorial diversificada. A todo lo anterior se suman dos requisitos fundamentales, el tener una cierta iniciativa internacional y una cierta capacidad para convertirse en actor, es decir poseer habilidad de movilizar recursos para la consecución de objetivos y capacidad de ejercer influencia sobre otros actores del sistema internacional (1996: 237).

Desde esta perspectiva, tomando la definición de actor político subestatal anteriormente descripta, las ciudades y regiones pueden ser consideradas como actores internacionales, aunque no sujetos de derecho internacional. Son considerados actores internacionales porque ejercen influencia y se han abierto al mundo accionando a través de diversas modalidades (acuerdos de relacionamiento, vinculaciones, hermanamientos, redes de incidencia regional y global, posicionamiento para atracción de inversiones y fomento del comercio, marketing de ciudades, entre otras).

Ahondando en el debate sobre actores, resulta interesante relevar la distinción aportada por Rosenau (1990) entre actores "libres de soberanía" (sovereignty-free) y actores "condicionados por la soberanía" (sovereignty-bound). Los autores como Hocking (1997) y Paquin (2005), en cambio, prefieren caracterizar a los gobiernos subnacionales como de categoría mixta. En ella se combinan el tener población, territorio e instituciones de gobierno. A partir de allí, se comparte soberanía con otros niveles de gobierno, lo que implica responsabilidades hacia una población, a la que se intenta satisfacer con el desarrollo de actividades internacionales.

En los estudios empíricos podemos observar que los gobiernos subnacionales llegan a asumir la defensa de intereses puntuales, a modos de grupos de interés, y a actuar en

\footnotetext{
${ }^{9}$ En esta definición se incluyen gobiernos locales, municipios, alcandías, áreas metropolitanas instituidas así como gobiernos provinciales de diverso tamaño, con distintas realidades socioeconómicas y con diferentes niveles de autonomía que actúan en RRII con diversos objetivos y motivaciones, (Zapata Garesché, 2007, p. 19).
} 
foros redes de ciudades mostrando comportamientos que pueden caer bajo la categoría de actores mixtos (Salomón, 2007: 6).

\section{B - $\quad$ Abordajes Desde La Política Exterior}

Dentro de los estudios sobre paradiplomacia se destacan los análisis que toman variables de orden doméstico como explicativas de su potenciación. Entre estas variables observadas adquieren relevancia las características del régimen de gobierno y el sistema político interno de las naciones. Los trabajos pioneros que abordaron estos determinantes internos fueron los de Duchacek (1990) y Soldatos (1990) en estudios sobre países centrales.

Los estudios que explican la acción exterior de ciudades y regiones centradas en la segmentación argumentan que la misma tuvo sus razones en la creciente burocratización, deshumanización y el alejamiento del Estado central de las entidades subnacionales. Todo lo anterior las habría llevado a actuar desde lo local a fin de hacer escuchar sus voces e intereses en el plano externo; lo cual es el elemento constitutivo de los incentivos de actuación externa en los análisis de política exterior (APE).

La regulación monopólica de asuntos sensibles y de interés para los gobiernos centrales fue cuestionada por gobiernos locales en temas como el comercio exterior; los fondos de cooperación; la generación de prestigio y visibilidad o la atracción de inversiones directas para sus territorios.

La desconcentración y deslocalización, fueron desarrolladas en la obra de Boisier (2010) y Cornago (2010). El proceso de redemocratización en la década del ochenta en América Latina posibilitó un mayor pluralismo de actores y procesos al interior de los sistemas políticos. Los casos estudiados para México (Schiavon, 2010); Brasil (Vigevani \& Prado, 2010) y (Milani \& Riveiro, 2010) y Argentina (Colacrai \& Zubelzu, 1994) aportan explicaciones en este sentido. Se abordan también las reformas constitucionales como centrales en la jerarquización y el reconocimiento jurídico de la posibilidad de acción internacional de gobiernos subnacionales. Tales son los casos de la reforma constitucional de Brasil de 1988 y de Argentina de 1994.

Uno de los desarrollos conceptuales que ha dado cuenta del fenómeno con mayor alcance y difusión es el de paradiplomacia acuñado por Duchacek (1990) y Soldatos \& 
Michelmann (1990). Allí se analiza el fenómeno como expresión de la crisis del Estado y la segmentación de la política exterior en países industrializados con sistemas federales, destacando la interrelación entre el sistema político doméstico e internacional.

Sin embargo, la utilización del concepto de paradiplomacia ha sido cuestionado por su sentido polisémico, es decir por la pluralidad de significados como expresión lingüística, así como por su falta de precisión metodológica (Ippolito, 2017). La paradiplomacia se refiere a las RRII desarrolladas por los gobiernos no centrales de un país - subestatales, regionales o locales - con el fin de promover sus propios intereses (socioeconómicos, culturales o medioambientales). Cada ente subestatal se proyecta a través de diversas modalidades (permanentes o ad hoc) realizando una diplomacia de acuerdo a su escala o posibilidades y considera que el accionar internacional subnacional puede desarrollarse dentro de sus competencias constitucionales (Cornago, 2010).

En sentido contrario al anterior, el concepto de protodiplomacia (Aldecoa \& Keating, 1999), describe situaciones de accionar externo subnacional en franca contraposición con el gobierno central y con una historia de reivindicaciones autonomista e incluso secesionista, como pueden ser los casos de Flandes y Quebec.

Los estudios de Maira y Calvento intentan ajustar el concepto a observaciones del fenómeno en contexto de América Latina. A partir de lo cual desarrollan aportes sobre el concepto de política internacional subnacional considerando que representa mejor a las particularidades de la región. Destacan la armonía y complementación de las políticas subnacionales con las políticas exteriores de los Estados centrales e incluso con los procesos de integración de la región latinoamericana (Maira, 2010) ${ }^{10}$.

Las investigaciones que problematizan la internacionalización de los gobiernos subnacionales como política pública local hacen hincapié en observar la existencia de una estrategia estable e instrumentos institucionales y formales generados a tal fin dentro de los gobiernos locales. "Algunos gobiernos subnacionales tienen una política

\footnotetext{
${ }^{10}$ En Argentina, el marco constitucional habilita a partir de la reforma de 1994 a la participación de las provincias, como entes subnacionales con competencias para suscribir convenios internacionales (art. 124 de la Constitución Nacional). No obstante, dichos acuerdos y convenios están subordinados a las prioridades y objetivos del Estado, es decir que la facultad de los actores subnacionales se limita a estipular lineamientos en dichos acuerdos a fin de satisfacer necesidades locales, pero siempre en el marco de la estrategia nacional.
} 
internacional subnacional en el sentido de que elaboran deliberadamente una estrategia destinada a alcanzar sus objetivos más allá de las fronteras y desarrollan instrumentos y estructuras institucionales para hacerlo. Es decir, diseñan e implementan una política en el sentido de un plan, opuesta a una práctica. Otros en cambio se limitan a una acción exterior, contactando o vinculándose con entidades extranjeras pero sin el respaldo de una estrategia exterior preconcebida, y con poca coordinación entre diferentes de gobierno" (Salomón, 2007:10).

Se inscriben en esta línea los estudios sobre internacionalización de gobiernos subnacionales que la abordan cómo la "decisión política y la herramienta pública de los gobiernos locales que se ocupa de impulsar la inserción internacional a través de una estrategia y objetivos tendientes a aprovechar, articuladamente, las oportunidades del contexto con las necesidades del territorio" (Calvento, 2016: 17). A lo anterior se suma la condición de estructurarse como una política transversal de la gestión pública local involucrando diversas áreas del gobierno (Milani \& Ribeiro, 2010).

El examen sistemático de la acción exterior de los gobiernos locales es posible realizarla desde la perspectiva del APE. Si bien tradicionalmente se ocupó del estudio de los factores determinantes, procesos de toma de decisiones e implementación de políticas exteriores de los Estados nación es dable que en la medida que actores diferentes al Estado van cobrando importancia se les preste cada vez más atención, siendo lícito aplicar herramientas conceptuales de dicha área de la disciplina a otros actores (Salomón, 2007:3).

El estudio de la acción subnacional ha promovido la apertura de múltiples líneas de investigación en el campo de las políticas exteriores. Incluso cuando existe gran cantidad de trabajos analíticos, estudios comparativos y de casos, no se ha logrado consenso respecto de un dilema básico: ¿en qué medida estas expresiones de accionar internacional subnacional constituyen o contribuyen políticas exteriores? (Luna Pont,2010). Esta última consideración remite a reconocer la necesidad de desplegar un programa de investigación en el campo de los estudios sobre subestatalidad en RRII que, anclados en estudios de política exterior, profundice el análisis de las diversas estrategias de inserción internacional subnacional (con actores de distintos tipos como 
megaciudades, ciudades grandes o intermedias, regiones metropolitanas, entre otros), contribuya a evaluar resultados e impactos de los diferentes tipos de estrategias y estudie su articulación con la diplomacia, el federalismo y la política exterior.

\section{C - $\quad$ La Integración Regional Y La Internacionalización De Actores Subnacionales}

Un lugar diferenciado tomarán los estudios sobre los procesos de integración regional, cuando ellos son abordados como propulsores de la actividad internacional subnacional. Los bloques de países integrados configuran espacios propicios para que los actores subnacionales busquen relacionarse internacionalmente, más aún cuando comparten fronteras o límites geográficos. Aquí podemos mencionar los antecedentes del proceso de construcción de la Unión Europea, mientras los Estados profundizaron la integración con iniciativas de traspaso de soberanía hacia instituciones comunitarias, los gobiernos regionales o subnacionales (regiones, Länders, Comunidades Autónomas, etcétera) demandaron cada vez mayor participación en la toma de decisiones.

En América Latina, el estudio del accionar de entes subnacionales dentro de las iniciativas de integración regional tiene su apogeo en la década de los ochenta y noventa. En el Cono Sur, las relaciones bilaterales entre Argentina y Brasil se transformaron e intensificaron a partir del retorno a la democracia. En el caso argentino las provincias fronterizas incrementaron su accionar externo como resultado de la integración regional a partir de los acuerdos de cooperación entre los presidentes Raúl Alfonsín y José Sarney. Es así que en 1985 se profundizó la integración bilateral y los gobiernos provinciales de Argentina que componen la CRECENEA (Comisión Regional del Nordeste Litoral) junto a los Estados del Sur de Brasil reunidos en el CODESUL (Conselho para o Desenvolvimento do Sul do Brasil), fueron incorporados oficialmente al Programa de Integración y Complementación Económica (PICE) a partir del Protocolo Regional Fronterizo $n^{\circ} 23$ (Colacrai \& Zubelzu, 1994).

El proceso de implementación del Mercosur fue produciendo instancias formales de participación de actores subnacionales. Tal es el caso de la creación de Mercociudades en 1995; luego vinieron el Foro Consultivo de Municipios, Estados Federados, Provincias y Departamentos del Mercosur (FCCR) en funcionamiento desde 2007 y posteriormente el Grupo de Integración Fronteriza (GIT). El aumento de los estudios sobre integración 
transfronteriza evidencia la riqueza del tema sobre política internacional subnacional. Estudios sobre el particular son los de la Triple Frontera entre Brasil, Argentina y Paraguay (Rhi-Sausi \& Oddone, 2010) o los referidos a la cooperación entre Chile y Argentina (Colacrai, 2010); (Silva Soura \& Morán León, 2010).

Como ya hemos mencionado en un apartado anterior, el pluralismo, el transnacionalismo y la interdependencia son las perspectivas más adecuadas para explicar los fenómenos internacionales que dan cuenta de la relación entre Estados y gobiernos subnacionales. El crecimiento de las interacciones económicas ha llevado al pluralismo a minimizar la preocupación por la seguridad y adoptar una definición del sistema internacional como un ámbito organizado a través del reconocimiento de intereses comunes y prácticas que emergen de la interdependencia. Asimismo, las RRII fueron concebidas como mediadas por actores, temas y sucesos ajenos a la voluntad de los Estados. Bajo este prisma la política exterior es resultado de un proceso en el cual una pluralidad de actores, como grupos de interés, las burocracias, los individuos intenta hace prevalecer sus intereses (Gámez, 2005: 134).

Los estudios focalizados en relaciones bilaterales entre países, fundamentalmente aquellas relaciones transfronterizas impulsadas por los procesos de integración, pueden ser ejemplos de abordajes que se posan sobre agendas múltiples, que comprenden un variado elenco de actores gubernamentales e issues areas para mostrar procesos, logros y déficits de las políticas desarrolladas. Aquí vemos cómo el estudio de las políticas exteriores, enmarcadas en procesos de integración regional que son propulsores de la actividad internacional subnacional, puede ser amplio y rico en categorías reflejando relaciones transgubernamentales, de múltiples voces o actores tanto públicos como privados o de la sociedad civil hasta llegar incluso a la "diplomacia de los partidos políticos" (Colacrai, 2016).

\section{D - $\quad$ Perspectivas Desde El Desarrollo Y Los Territorios}

Desde otro subconjunto de estudios los enfoques de la EPI en combinación con la Nueva Geografía Económica, la Economía del Desarrollo y los Estudios Urbanos articulan territorialidad y desarrollo. Estas perspectivas enfocan su atención en los cambios operados en el papel del espacio/territorio en el marco de un proceso de globalización. 
Se analiza la función del territorio como factor central en la relación entre las sociedades y el mercado global en el marco de importantes mutaciones operadas en la capacidad mediadora del Estado. Lo anterior explica, en parte, la búsqueda de oportunidades para maximizar la capacidad de acción subnacional dentro del estado, a nivel regional e internacional y en relación con diversas redes y dinámicas internacionales (Luna Pont, 2010) .

Las diferentes vertientes teóricas de la EPI, producto del debate neo-neo mencionado anteriormente, han aportado a la comprensión de las fuerzas que operan y explican el desempeño de actores subestatales. El estudio sobre coaliciones, grupos de interés, lobbies, burocracias de los distintos niveles de gobierno como agentes que buscan maximizar su utilidad o satisfacción en un contexto dado de incentivos y restricciones derivados del proceso de globalización son aplicados a estudios sobre subestalidad en RRII.

Desde el punto de vista geográfico, una de las mayores consecuencias de la globalización fue despojar de centralidad a la escala nacional de acumulación, urbanización y regulación estatal. Esta redefinición de la escala en que se realiza la dinámica del capital y un nuevo diseño territorial es denominada "glocalización"11. Lentamente comienza un proceso de revalorización de las escalas geográficas supranacionales y subnacionales como ámbitos específicos de interacciones sociales, procesos políticos y de relaciones de poder y las categorías analíticas se amplían y resignifican el concepto de desarrollo.

Surge en dicho contexto la perspectiva endógena del desarrollo. Ella pondera el rol que asumen los actores locales, el tipo de interacciones que éstos llevan a cabo y las características del entorno de actuación, como componentes estratégicos fundamentales del mismo. Aquellos que valorizan las virtualidades de este fenómeno orientan sus análisis e investigaciones hacia los procesos locales y regionales rescatando la amplia y singular relación entre las características de cada ámbito subnacional -en especial las ciudades medias y grandes y las redes de ciudades de menor dimensión- y

\footnotetext{
${ }^{11}$ El término fue acuñado por Robert Robertson, refiere a un proceso combinado de globalización y reconfiguración local territorial, y a un proceso de desterritorialización y reterritorialización que ha afectado las relaciones entre ciudades y estados como modos interrelacionados de organización socio-económica, política y geográfica.
} 
los procesos más agregados de desarrollo y presumen que las dinámicas de crecimiento y acumulación de capital han bajado de escala.

La construcción de una política de desarrollo local requiere de la integración de visiones e intereses, y el territorio no es visto solo como lugar de identidad sino también como construcción política no exenta de conflictos y realidades de poder. Este enfoque entiende que el desarrollo se ha convertido en un fenómeno fuertemente localizado, que se debe organizar, planificar y gestionar desde cada unidad territorial con capacidades de decisión estratégica, como son las ciudades y los espacios regionales (Madoery, 2016). En el marco de dicho proyecto han emergido políticas públicas locales innovadoras, entre ellas se destacan las estrategias de internacionalización, que buscan por un lado, hacer frente a nuevas exigencias del contexto exógeno y, por otro, aprovechar las nuevas condiciones para el mejoramiento de la gestión pública local. El desarrollo no es concebido como un fenómeno de carácter local sino un proceso complejo que requiere una nueva geografía de responsabilidades públicas, de un estado activo en todas sus escalas (nacional, provincial, local), de la conformación de redes de ciudades y de la compensación y equilibrio entre ciudades y regiones ya que el contexto general no afecta a todos los territorios de la misma manera (Madoery; 2003).

Luego de revisar y reseñar los diversos subconjuntos de enfoques sobre lo subnacional en las RRII volvemos a citar a Halliday; quien nos hace reflexionar al sostener que en el plano teórico la disciplina reproduce en cierto modo la diversificación y la fragmentación que caracteriza a otras disciplinas como la sociología, la geografía y la historia. Y agrega que "aunque el principio de que "todo vale" es peligroso, es deseable una situación de pluralismo teórico siempre que cada una de las teorías genere una agenda de investigación que desemboque en un análisis sustancial" (2006:21). Sostenemos que dada la relativa novedad del fenómeno observado dentro de la disciplina y lo heterogéneo de las situaciones analizadas, resulta natural que la mayor parte de la literatura sobre actores subnacionales sea de carácter descriptivo. Sin embargo destacamos y afirmamos que existe una comunidad de investigación científica con desarrollos y elaboraciones conceptuales logradas, a partir de la cual es posible la 
construcción de una genuina agenda de investigación que le de sustento y que potencie el campo en el mediano plazo.

\section{4- CONSIDERACIONES FINALES}

A través del presente trabajo nos propusimos recorrer las perspectivas teóricas dentro de la disciplina desde las cuales han surgido categorías analíticas aplicadas al campo de estudios sobre la internacionalización de ciudades y regiones. Este último se caracteriza por su fragmentación y la pluralidad de abordajes en el estado actual de la producción; aunque también se encuentra nutrido desde los "grandes debates" teóricos de las RRII y muy fuertemente por el pluralismo de las teorías de alcance medio.

De la revisión realizada constatamos la recurrencia del eclecticismo teórico en los estudios sobre subestatalidad en RRII en los últimos años. La proliferación de combinaciones de categorías teóricas en los análisis resulta un medio que ayuda a lograr explicaciones multidimensionales a fenómenos complejos. Lo anterior se observa mayormente en las investigaciones que se sostienen desde las teorías de alcance medio; tal es el caso de los análisis de la política exterior. Consideramos que será necesario que el subcampo de estudios sobre subestatalidad logre producir agendas propias y mayores análisis rigurosos a fin de legitimarse como comunidad de conocimiento.

Hemos destacado las contribuciones a partir del tercer debate teórico de las RRII que incluye al transnacionalismo y la teoría de la interdependencia compleja con los realistas. Si bien dicho debate no trata la temática de la subestatalidad en forma directa se reconocen innovaciones conceptuales que amplían los marcos de análisis sobre el fenómeno bajo estudio. El recorrido por el debate entre los neorrealistas y los neoliberales muestra que sus resultados se han plasmado fructíferamente en nuevos enfoques y categorías. En este sentido, la EPI y el concepto de régimen internacional han aportado a la comprensión de la internacionalización de gobiernos locales. En particular en aquellos abordajes que relacionan internacionalización con desarrollo y territorio, así como los que revisan la participación de actores subnacionales en asociaciones y redes de ciudades a nivel global.

Las líneas de investigación que abordan las causas de la internacionalización de entidades subnacionales se pueden clasificar desde una doble perspectiva. Por un lado, 
algunas explicaciones ponen el acento en la dinámica y estructura del sistema internacional, que podría jugar como variable dependiente. Por otro lado, aquellos análisis sobre política exterior que hacen hincapié en las estructuras o factores internos como factores explicativos del accionar internacional de gobiernos locales. Estos últimos aportaron la idea de que los actores y factores domésticos son relevantes para el contenido y la formulación de la política externa y que dependería del "cuándo" (el contexto histórico) y el "dónde" (el área temática específica) para definir su peso como variable explicativa.

En los últimos tiempos ha habido un gran esfuerzo de producción y sistematización de estudios y compilaciones sobre internacionalización de ciudades y regiones en la disciplina. Todos aportan y enriquecen el debate y la reflexión que se encuentra vivo y dinámico y está muy lejos de ser conclusivo. De lo anterior hemos podido reseñar y presentar cuatro grandes subconjuntos de abordajes sobre el tema. Aquellos centrados en la perspectiva del actor internacional; quienes lo abordan desde el análisis de políticas exteriores; los que lo hacen desde las teorías de la integración regional y, finalmente perspectivas que articulan la relación entre territorialidad y el desarrollo.

En la mirada que privilegia los debates sobre el actor internacional se destacan visiones sobre la gravitación de empresas multinacionales, de ONGs y la emergencia de nuevos movimientos sociales, redefiniendo los conceptos y las tipologías. Las categorías creadas reflejarían un mundo visto como aldea global, policéntrica y de soberanías perforadas.

Dentro de los análisis de política exterior y el ajuste del nivel de análisis pueden lograrse gradualidades y sutilezas en las contribuciones que reflejen las transformaciones del sistema político interno. Lo anterior habilita la mirada sobre la toma de decisiones de la burocracia, las tradiciones, el liderazgo y los ajustes entre lo interno y externo. La observación y estudio de casos de internacionalización nos acerca a un abordaje que, anclados en estudios de política exterior, profundice el análisis de los impactos y resultados de las diversas estrategias de inserción internacional subnacional y estudie su articulación con la diplomacia, el federalismo y la política exterior. 
Los bloques de países integrados configuran espacios propicios para que los actores subnacionales se internacionalicen. Para América del Sur el proceso de creación e implementación del Mercosur produjo instancias formales de participación de actores subnacionales como es Mercociudades y el Foro Consultivo de Municipios que nutren estudios del campo.

Los abordajes desde la EPI vinculan desarrollo y territorio y complementan aquellos sobre subestatalidad. Los estudios sobre desarrollo endógeno, la generación de capacidades de desarrollo en los territorios que explican la internacionalización se inscriben en dicho eje. Se observan cómo los proyectos políticos locales, que sintetizan la relación entre política y territorio, son propulsores de la emergencia de políticas públicas locales innovadoras. En esta categoría se pueden contar las estrategias de internacionalización de actores subnacionales, que buscan, por un lado, hacer frente a nuevas exigencias del contexto exógeno y, por otro, aprovechar las nuevas condiciones para el mejoramiento de la gestión pública local.

Desde los años setenta los grandes debates teóricos de la disciplina de las RRII han contribuido con conceptos, categorías analíticas y metodológicas a ampliar y enriquecer el campo de estudios sobre la internacionalización de ciudades y regiones y, en el siglo XXI nos muestran una proliferación de estudios basados en el eclecticismo teórico y sostenido por teorías de alcance medio.

Es en este escenario en el cual las investigaciones y estudios se caracterizan por el pluralismo metodológico, acercamientos desde la historia y desde el estudio de casos así como el análisis de grandes conjuntos de datos en sus observaciones. Priorizan la contingencia histórica y focalizan sus estudios en una parte del proceso político, no intentan abarcarlo en su totalidad y estudian los efectos de una o varias variables de decisión política así como sus resultados. Lo anterior incluye la revalorización de lo doméstico en las RRII tal como lo sostienen los estudios que incorporan la economía política internacional. Promovemos la articulación de visiones teóricas como herramientas conceptuales y analíticas a fin de enriquecer los abordajes e investigaciones a futuro, siempre respetando y cuidando la coherencia interna del 
eclecticismo, para la compresión del fenómeno multidimensional de la subestatalidad en la disciplina de las relaciones internacionales.

\section{REFERENCIAS BIBLIOGRÁFICAS}

Arenal, C. (1984). 'La teoría de las relaciones internacionales hoy: debates y paradigmas', Revista Estudios Internacionales, 22(86), Chile. pp. 153-182

Batista, S.; Jakobsen, K.; Evangelista, A. (2008). 'La apertura al exterior de las ciudades latinoamericanas y la cooperación descentralizada', Observatorio de cooperación descentralizada Unión Europea-América Latina. Montevideo, Editorial: Observatorio de cooperación descentralizada UE-AL.

Boisier, S. (2010). 'Un marco para la paradiplomacia desde las estrategias de desarrollo regional: el retorno del actor territorial en un nuevo escenario', In: Maira, L. La política internacional subnacional en América Latina. Buenos Aires: Libros del Zorzal, pp 39-62.

Calvento, M. (2016). 'Procesos y actores en la gestión de la política internacional subnacional', pp. 15-40. Tandil: CEIPIL.

Colacrai, M. (2010). 'El tejido de relaciones bilaterales desde el ámbito subnacional. Desarrollo de una innovadora interdependencia entre la Argentina y Chile', In: Maira, L. La política internacional subnacional en América Latina. Buenos Aires: Libros de Zorzal, pp. 305-328.

. (2016). 'La relación bilateral Argentina-Chile en clave política 2010-2015. Continuidades y matices con los cambios de gobierno', Instituto de Estudios Internacionales, Universidad de Chile, pp. 9-37.

Colacrai, M.; Zubelzú, G. (1994). Las provincias y sus relaciones externas: ¿Federalización de la política exterior o protagonismo provincial de las relaciones internacionales?. Rosario: Cerir.

(2004). Las vinculaciones externas y la capacidad de gestión internacional desplegadas por las provincias argentinas en la última década: Una lectura desde las relaciones internacionales. Buenos Aires, CARI.

Cornago, N. (1999). 'Diplomacy and paradiplomacy in the redefinition of international security: dimension of the conflict and cooperation', In: Keating, M.; Aldecoa, F. 
Paradiplomacy in Action: The Foreign relations of subnational governments. Portland: Frank Cass Publishers, pp. 40-57.

- (2010). 'La descentralización como elemento de innovación diplomática: aproximación a sus causas estructurales y lógicas de acción', In: Maira, L. Política internacional subnacional en América Latina. Buenos Aires: Libros del Zorzal, pp. 107134.

Duchacek, I. (1990). 'Perforated sovereignties: towards a tipology of new actors in international relations', In: Soldatos, P.; Michelmann, H. J. Federalism and International Relations: The role of subnational units. Oxford: Clarendon Press.

Gámez, A. (2005). 'Fuentes de cambio en política exterior. Una revisión de los modelos de política exterior para los países en desarrollo', Revista CIDOB d'Afers Internacionals, pp. 127-151.

Garcia Segura, C. (1996). 'La actividad exterior de las entidades políticas subestatales', Revista de Estudios Políticos (Nueva Epoca), pp. 235-264.

Halliday, F. (2006). 'Las Relaciones Internacionales y sus debates', Informe Centro de Investigación para la Paz (CIP-FUHEM). Madrid.

Ippolito, D. (2017). 'Relaciones internacionales de gobiernos no-centrales: un análisis teórico-conceptual de la paradiplomacia y de sus variables explicativas', Cuadernos de Política Exterior Argentina (Nueva Época), pp. 29-47. Rosario: CERIR.

Keohane, R. (2012). 'Twenty years of institutional liberalism', International Relations, 26(2), pp. 125-138.

; Nye, J. (1988). Poder e interdependencia. La política mundial en transición.

Buenos Aires: Grupo Editor Latinoamericano.

Sodupe, K. (2003). La teoría de las Relaciones Internacionales a comienzos del Siglo XXI. Guipúzcoa: Servicio Editorial de la Universidad del País Vasco.

Lachapelle, G.; Paquin, S. (2005). Mastering Globalization: new subnational governance and strategies. Londres: Routledge. 
Lake, D. (2013). 'Theory is dead, long live theory: The end of the Great Debates and the rise of eclecticism in International Relations', European Journal of International Relations, 19(3), pp. 567-587.

Lara Pacheco, R. (2019). La inserción de las ciudades en el medio internacional. Una revisión histórica, teórica y empírica desde las relaciones internacionales. México: Universidad de Guadalajara - Centro Universitario de Ciencias Económico Administrativas.

Lee Ray, J. (2001). 'Integrating levels of Analysis in World Politics', Journal of Theoretical Politics, pp. 355-388.

López, M.; Oddone, N. (2010). Las ciudades y los poderes locales en las relaciones internacionales contemporáneas. España: UIM.

Luna Pont, M. (2010). 'Perspectivas teórico-conceptuales de las ciudades y los poderes locales dentro de la dimensión subnacional de las RRII', In: Lopez, M.; Oddone, N. (Comps.) Las ciudades y los poderes locales en las relaciones internacionales contemporáneas. España: UIM, pp. 49-78.

(2011). 'Las relaciones internacionales subnacionales desde la teoría de las relaciones internacionales', Trabajos de Investigación en Paradiplomacia, pp. 43-64.

Madoery, O. (2003). 'La primera generación de políticas locales de desarrollo en la Argentina: contexto, características y desafíos', Centro de Estudios del Desarrollo y territorio. Universidad de San Martin.

(2016). Los desarrollos latinoamericanos y sus controversias. Ushuaia: Editorial UNTDF.

Maira, L. (2010). La Política Internacional Subnacional en América Latina. Buenos Aires: Libros del Zorzal.

Martin, P. (2010). 'La cooperación internacional al desarrollo de las ciudades y los poderes locales', In: Lopez, M.; Oddone, N. (Comps.) Las ciudades y los poderes locales en las relaciones internacionales contemporáneas, pp. 173-187. España: UIM 
Marx, V. (2010). 'Las ciudades y su inserción en las RRII', In: Lopez, M.; Oddone, N. (Comps.) Las ciudades y los poderes locales en las relaciones internacionales contemporáneas. España: UIM, pp. 28-46.

Milani, M.; Ribeiro, C. (2010). 'Paradiplomacia y proyección internacional de las ciudades brasileñas: la elaboración del concepto de "gestión internacional local", Revista Geopolítica(s), 1(1), pp. 23-40.

Oddone, N. (2016). 'La paradiplomacia desde cinco perspectivas: reflexiones teóricas para la construcción de una comunidad epistémica en América Latina', Revista Relaciones Internacionales, Universidad Nacional de Costa Rica, 89(2), pp. 47-81

Rhi-Sausi, J.; Oddone, N. (2010). 'Cooperación e integración transfronteriza en el Mercosur: el caso de la Triple Frontera entre Argentina, Brasil y Paraguay', In: Maira, L. Política internacional subnacional en América Latina. Buenos Aires: Libros del Zorzal, pp. 209-258.

Romero, M. (2004). Una Aproximación Contextual y Conceptual a la Cooperación Descentralizada. Valparaíso: Municipalidad de Valparaíso y Diputación de Barcelona.

Rosenau, J. (1990). Turbulence in Word Politics. Princenton: Princenton University Press.

Russell, R. (1986). 'Teorías contemporáneas en relaciones internacionales', In: Russell, R. Temas de política exterior latinoamericana: El caso uruguayo. Grupo Editor Latinoamericano.

(2010). 'El estado nación y los actores gubernamentales no centrales: una relación complementaria', In: Maira, L. Política internacional subnacional en América Latina. Buenos Aires: Libros del Zorzal, pp. 83-106.

Salomón González, M. (2002). 'La teoría de las Relaciones Internacionales en los albores del siglo XXI: diálogo, disidencia, aproximaciones', CIDOB d'Afers Internacionals, pp. 752.

(2007). 'La acción exterior de los Gobiernos subnacionales y el análisis de políticas exteriores', $1^{\circ}$ Encontro Nacional de ABRI Politica Externa, pp. 1-17. 
Schiavon, J. (2010). 'Las relaciones exteriores de los gobiernos estatales: el caso de México', In: Maira, L. Política internacional subnacional en América Latina. Buenos Aires: Libros del Zorzal, pp. 135-176.

Silva Soura, V.; Morán León, C. (2010). 'Relaciones subnacionales. El caso de Chile y Argentina', In: Maira, L. Política internacional subnacional en América Latina. Buenos Aires: Libros del Zorzal, pp. 259-304.

Sodupe, K. (2003). La teoría de las RRII a comienzos del siglo XXI. Universidad del País Vasco: Servicio Editorial Bilbao.

Soldatos, P.; Michelmann, H. (1990). Federalism and International Relations: The role of subnational units. Oxford: Clarendon Press.

Strange, S. (1988). States and Markets. London: Pinter Publishers Limited

Vigevani, T.; Prado, F. B. (2010). 'Acciones y problemas de la paradiplomacia en Brasil', In: Maira, L. Política internacional subnacional en América Latina. Buenos Aires: Libros del Zorzal, pp. 177-208.

Zapata Garesché, E. (2007). Manual práctico para internacionalizar la ciudad. Guía para la acción exterior de los gobiernos locales y la cooperación descentralizada Unión Europea-América Latina. Barcelona: Diputación de Barcelona.

Zeraoui, Z. (2016). 'Para entender la paradiplomacia', Revista Desafíos, 28(1), pp. 15-34. 